\title{
Erratum to: A Preliminary Investigation of the Spence Children's Anxiety Parent Scale as a Screening Tool for Anxiety in Young People with Autism Spectrum Disorders
}

\author{
Hani Zainal · Iliana Magiati · Julianne Wen-Li Tan • \\ Min Sung • Daniel S. S. Fung • Patricia Howlin
}

Published online: 26 March 2014

(c) Springer Science+Business Media New York 2014

\section{Erratum to: J Autism Dev Disord}

DOI 10.1007/s10803-014-2075-0

Unfortunately, one of the co-author, Patricia Howlin's affiliations was incorrectly entered in the original publication of the article. The corrected affiliations are presented with this erratum.

The online version of the original article can be found under doi:10.1007/s10803-014-2075-0.

H. Zainal · I. Magiati $(\bowtie) \cdot$ J. W.-L. Tan

Department of Psychology, National University of Singapore

(NUS), AS4, 9 Arts Link, Singapore 117570, Singapore

e-mail: psyim@nus.edu.sg

M. Sung · D. S. S. Fung

Department of Child and Adolescent Psychiatry, Institute of

Mental Health, Buangkok, Singapore

P. Howlin

Institute of Psychiatry, King's College London, London, UK

P. Howlin

Faculty of Health Sciences, University of Sydney,

Sydney, NSW, Australia 